\title{
Diagnosing Graves' Disease and Non-Graves Hyperthyroidism Using TSH Receptor Antibody Test versus Non-TSH Receptor Antibody Test Methods of Diagnosis
}

\author{
Mohammed Qader Meena $(\mathbb{0}$ \\ Department of Medicine, College of Medicine, Hawler Medical University, Erbil, Iraq \\ Email:mmeneh@gmail.com
}

How to cite this paper: Meena, M.Q. (2020) Diagnosing Graves' Disease and Non-Graves Hyperthyroidism Using TSH Receptor Antibody Test versus Non-TSH Receptor Antibody Test Methods of Diagnosis. Open Journal of Endocrine and Metabolic Diseases, 10, 7-17.

https://doi.org/10.4236/ojemd.2020.102002

Received: January 22, 2020

Accepted: February 14, 2020

Published: February 17, 2020

Copyright $\odot 2020$ by author(s) and Scientific Research Publishing Inc. This work is licensed under the Creative Commons Attribution International License (CC BY 4.0).

http://creativecommons.org/licenses/by/4.0/

\begin{abstract}
Background: Differentiating Graves hyperthyroidism from the other causes of hyperthyroidism, using serum TRAb testing is essential step for diagnosis. Objectives: To study importance of TRAb in the diagnosis of Graves' disease, distinguishing it from thyroiditis, and comparing it with clinical features and other tests such as TPOAb, US thyroid and thyroid scintiscan. Methods: A cross-sectional study was conducted on 120 patients attending endocrine clinicErbil city. Patients were studied on clinical feature basis and investigated with serum TRAb, TPOAb, TSH, Free T4, and Ultrasound examination of thyroid gland. Fisher exact test and Chi Square test of independence, Correlation coefficient and t-test of independence were used. Results: Fifty-two patients were found to have Graves' disease; There was significant correlation between TRAb positivity and diagnosis of Graves' disease $p<0.05$. There was also strong relation between presence of goiter, Orbitopathy and Ultrasound findings and TRAb positivity, $\mathrm{p}<0.05$. There was statistically significant difference between mean levels of TRAb and fT4 between Graves patients and Thyroiditis groups, while there was no statistically significant difference in TPOAb and TSH means between the two groups, $\mathrm{p}>0.05$. Conclusion: A positive correlation was found between TRAb titer and positivity and no significant relation between TPOAb levels between Graves' disease patients compared with thyroiditis patients, respectively.
\end{abstract}

\section{Keywords}

Graves' Disease, Thyrotoxicosis, Toxic Multinodular Goiter (TMNG), Thyroiditis, TSH Receptor Antibodies (TRAb) 


\section{Introduction}

Thyrotoxicosis is a clinical state resulting from inappropriately high thyroid hormone levels [1].

Endogenous hyperthyroidism is most commonly due to GD or nodular thyroid disease. GD is an autoimmune disorder in which thyrotropin receptor antibodies (TRAb) stimulate the TSH receptor, increasing thyroid hormone production and release. The development of nodular thyroid disease includes growth of established nodules, new nodule formation, and development of autonomy over time [2].

Although toxic nodular goiter is less common than GD, its prevalence increases with age and in the presence of dietary iodine deficiency. Therefore, toxic nodular goiter may actually be more common than GD in older patients, especially in regions of iodine deficiency [3] [4]. Unlike toxic nodular goiter, which is progressive (unless triggered by excessive iodine intake), remission of mild GD has been reported in up to $30 \%$ of patients without treatment [5].

Less common causes of thyrotoxicosis include the entities of painless and subacute thyroiditis, which occur due to inflammation of thyroid tissue with release of preformed hormone into the circulation.

The etiology of thyrotoxicosis should be determined. If the diagnosis is not apparent based on the clinical presentation and initial biochemical evaluation, diagnostic testing is indicated and can include, depending on available expertise and resources: 1) measurement of TRAb, 2) determination of the radioactive iodine uptake (RAIU), or 3) measurement of thyroidal blood flow on ultrasonography. A $123 \mathrm{I}$ or $99 \mathrm{mTc}$ pertechnetate scan should be obtained when the clinical presentation suggests a TA or TMNG [1].

It is caused by the presence of autoantibodies to the thyrotropin [TSH] receptor leading to unregulated production and secretion of thyroid hormones [6]. Typically, GD is characterized by suppressed serum TSH and overproduction of thyroid hormones thyroxine and triiodothyronine (T4 and T3) [6].

The diagnosis of GD is made on the basis of typical clinical features of hyperthyroidism, such as weight loss, fatigue, heat intolerance, tremor, palpitations and diffuse thyroid enlargement, plus specific clinical features of GD including orbitopathy, thyroid dermopathy (pretibial myxedema) and thyroid acropachy [7]. Serum analyses typically show suppressed thyroid-stimulating hormone (TSH; thyrotropin) and elevated thyroid hormones, tetraiodothyronine (T4; thyroxine) and triiodothyronine (T3) [8]. Additional diagnostic tests can include imaging (commonly ultrasound and radioisotope uptake study) and thyroid autoantibodies, which can help to distinguish GD from other causes of thyrotoxicosis.

The autoimmune production of TSH receptor antibodies (TRAb) is central to the pathogenesis of GD [7]. More recent American Thyroid Association (ATA) guidelines place greater emphasis on the use of TRAb, particularly in monitoring the course of Graves' disease, but similarly recommend that its place is in the diagnosis of GD in those patients whose etiology of hyperthyroidism is unclear from their presentation and biochemistry [1]. 
In most cases the diagnosis of Graves' disease can be made with sufficient certainty based on clinical presentation of the patient. However, TRAbs can be useful to establish the diagnosis and guide management in ambiguous clinical scenarios such as the differential diagnosis of painless thyroiditis, unilateral exophthalmos, euthyroid Graves' ophthalmopathy), subclinical hyperthyroidism and thyrotoxicosis associated with hyperemesis gravidarum [1]. TRAbs may also be useful in predicting the risk of recurrence of Graves' disease following a course of antithyroid drug treatment [2].

The thyrotropin receptor (TSHR) is a major autoantigen in autoimmune Hyperthyroidism. And specific autoantibodies acting as TSHR agonists (TRAb) are pathogenic (i.e. responsible for clinical manifestations) and are the diagnostic hallmark of Graves' disease (GD) [9]. Measurement of TRAb plays a crucial role in the differential diagnosis of hyperthyroidism [10].

\section{Materials/Methods}

A cross sectional study was conducted on 120 patients presenting with clinical features of thyrotoxicosis, in Endocrinology clinic, Erbil Teaching Hospital in Erbil City from December 2018 to October 2019. After complete history taking in relation to thyroid status and thorough examination for general clinical features of hyperthyroidism, goiter, evidence of orbitopathy and acropathy and thyroid status examination, the serum of all the patients were sent for necessary serum tests, including TSH (Thyroid Stimulating Hormone), Free T4 (FT4), and TRAb (TSH receptor antibodies) and TPOAbs (Thyroid peroxidase antibodies) for patients who were suspected of having Graves' disease or thyroiditis. The criteria for diagnosis of Graves' disease include clinical features of hyperthyroidism with Ophthalmopathy, Dermopathy or thyroid acropathy, TRAb Antibodies, 123 radioactive iodine uptake or 99 Technetium pertechnicate scans which shows increased diffuse uptake, and ultrasound scan of thyroid gland which shows enlarged gland with smooth contour and heterogeneous echotexture, and color doppler study of thyroid gland which shows diffuse increase vascularity of the thyroid gland.

The diagnostic criteria for thyroiditis was based exclusion of Graves hyperthyroidism, presence of high titers of autoantibodies mainly TPOAbs or thyroglobulin antibodies, as in cases of postpartum thyroiditis presenting with features of thyrotoxicosis; and ESR (Erythrocyte sedimentation Rate) and CRP (C-Reactive protein) as in case of Subacute (De Quervian) thyroiditis, ultrasound of thyroid gland which are non-specific, and radioactive uptake thyroid scans. ESR (Erythrocyte sedimentation Rate) and CRP were sent for patients suspected of having painful subacute thyroiditis (De Quervain thyroiditis). Ultrasound examination of thyroid gland were done for all the patients, to inspect for evidence of diffuse thyroid enlargement, as in case of Graves' disease and thyroiditis, or for thyroid adenoma and multinodular thyroid enlargement in Toxic multinodular goiter and toxic solitary adenomas. Doppler ultrasound of thyroid gland was not obtained for all the patients, because of technical unavailability, for all the patients who sus- 
pected to have Graves' disease, for that, it was excluded in the study. We couldn't include Thyroid scan for our patients in the study, because of unavailability of the test in our locality. Few patients (10 cases), had $99 \mathrm{mTechnitium} \mathrm{pertechnicate}$ scans available, were excluded in the study. Since T3 or Free T3 test was not routinely requested for all the patients in our study, it was excluded in the study.

Elecsys ${ }^{\circledR}$ Anti-TSHR were used for estimating plasma or serum levels of TSH Receptor Antibodies (TRAB). Elecsys Anti-TSHR (TRAK) is a fully automated test for detection of autoantibodies to the TSH receptor. COBAS Elecsys ${ }^{\circledast}$ anti-TPO were used for assessing TPO Antibodies. Elecsys ${ }^{\circledast}$ Thyrotropin (TSH) Assay were used to estimate serum levels of Thyroid stimulating Hormone (TSH).

The electrochemiluminescence immunoassay "ECLIA" is intended for use on Elecsys and Cobas e immunoassay analyzers. The high sensitivity of $97 \%$ and high specificity of $99.5 \%$ of the Elecsys Anti-TSHR assay support clinical accuracy. Free T4 (FT4) were assessed using Roche Cobas Laboratories Elecsys FT4 II Immunoassay for the in vitro quantitative determination of free thyroxine in human serum and plasma.

Non-Graves thyroid diseases were diagnosed on the basis of clinical symptoms, biochemical confirmation of hyperthyroidism, and additional diagnostic results, such as diffuse and/or nodular goiter, thyroid ultrasound, and other tests for diagnosis of thyroiditis. The study was approved by ethics committee, college of Medicine, Hawler Medical University. Informed verbal consent was taken from all the patients included in the study.

\section{Stastical Analysis}

Statistical analysis Data analyses were conducted using theStatistical Packages for the Social Sciences version 24.0 (IBM). Data were summarized using frequency tables, mean, and standard deviation. Chi square, Fisher's exact test were used stastical analysis. Correlation coefficient were used between different continuous and $t$ test was used for comparison between the means of continuous variables. The level of statistical significance was considered as $\mathrm{p}<0.05$.

\section{Results}

The study was done on 120 patients who presented with clinical features of thyrotoxicosis.

The age of the patients at the time of diagnosis was a mean \pm standard deviation $37.97 \pm 12.002$ (range: $16-78$ ) years; $83(69.2 \%)$ of patients were women and $37(30.8 \%)$ were men. History of thyroid status were taken from all the patients, and all the patients were examined physical signs of thyroid illnesses and thyroid status of the patients, including weight loss, goiter, and signs of orbitopathy, dermopathy (Pretibial Myxedema) and Graves Acropathy, which are specific features of Graves' Disease.

79 (65.8\%) of 120 patients, had weight loss (not quantified), 89 (74.2\%) had goiter (the clinical types of goiter were not recorded in the study), 24 (20\%) of cases had signs of Orbitopathy. 88 patients (73.3\%) had ultrasound findings of thyroid 
enlargement; 67 (55.8\%) had diffuse enlargement of thyroid gland and 29 (24.2\%) had multinodular US findings or had solitary nodule.

The patients were sub divided into three groups for final diagnosis: Group (1): patients diagnosed as Graves' disease; Group (2): patients diagnosed as having Toxic Multinodular Goiter and Toxic solitary Nodules, and Group (3): Thyroiditis including subacute painful (de Quervain) thyroiditis, chronic painless thyroiditis, postpartum thyroiditis and other causes of thyroiditis.

38 (31.7\%) of cases had elevated TSH-R Ab (TRAb) of more than $1.75 \mathrm{mU} / \mathrm{L}$, which was considered as cut-off value of positivity for the test.

The mean serum level of TSH, FT4, TRAb and TPOAb of all subjects diagnosed as having Graves' disease and Thyroiditis groups was shown in Table 1. Values are presented as mean \pm SD or number (\%) unless otherwise indicated.

Table 1. Baseline characteristics of Hyperthyroid patients and subgroups patients.

\begin{tabular}{|c|c|c|c|c|c|}
\hline Total cases/normal reference ranges & Number (\%) & Minimum & Maximum & Mean & Mean standard Deviation \\
\hline (Gender Total) & $120(100 \%)$ & & & & \\
\hline Female & $83(69.2 \%)$ & & & & \\
\hline Male & $37(30.8 \%)$ & & & & \\
\hline Age (years), mean $\pm \mathrm{SD}$ & [1] & 16 & 78 & 37.88 & 11.926 \\
\hline $\operatorname{TRAb}(0.9-1.25 \mathrm{IU} / \mathrm{L})$ & & & & 4.95 & 7.709 \\
\hline $\mathrm{TSH}(0.27-4.2 \mathrm{mIU} / \mathrm{L})$ & & & & 0.0915 & 0.22558 \\
\hline $\begin{array}{c}\text { fT4 }(9-22 \mathrm{pm} / \mathrm{L}) \\
\text { TPOAb }(>35 \mathrm{IU} / \mathrm{ml})\end{array}$ & & & & 22.5058 & 9.06363 \\
\hline Graves' disease Gr. & $52(43.3 \%)$ & & & & \\
\hline Age & & 16 & 63 & 35.3654 & 10.29188 \\
\hline TRAb & & 1 & 40 & 10.3773 & 8.90992 \\
\hline TSH & & 0 & 0.3 & 0.024 & 0.05518 \\
\hline $\mathrm{fT} 4$ & & 7 & 140 & 30.6538 & 30.05412 \\
\hline TPOAb & & 14 & 58 & 25.5871 & 9.39161 \\
\hline Thyroiditis Gr. & $39(32.5 \%)$ & & & & \\
\hline Age & & 16 & 51 & 33.6667 & 8.72785 \\
\hline TRAb & & 0 & 18 & 1.1397 & 2.8129 \\
\hline TSH & & 0 & 1.7 & 0.1563 & 0.3352 \\
\hline fT4 & & 11 & 58 & 20.8628 & 9.83407 \\
\hline TPOAb & & 5 & 500 & 64.1026 & 103.95287 \\
\hline Toxic Nodular Gr. & $29(24.2 \%)$ & & & & \\
\hline Age & & 27 & 78 & 48.5357 & 12.79607 \\
\hline TSH2 & & 0 & 1 & 0.1299 & 0.20917 \\
\hline FT2 & & 12 & 33.92 & 19.09 & 5.00586 \\
\hline TNG (n) & & 28 & & & \\
\hline
\end{tabular}

Abbreviation: TSH, Thyroid Stimulating Hormone; FT4, Free T4; TRAb, Thyrotropin Receptor Antibody; TPOAb, Thyroid Peroxidase Antibodies. 
In the study we found strong association between serum TRAb levels and gender, presence of Orbitopathy, goiter, and ultrasound findings of thyroid gland (Table 2).

There is strong correlation between TRAB concentrations and levels of TSH, FT4 and TPO Antibodies < 0.05, and there was strong relation between TSH, FT4, TRAB and TPOAb levels while there was no significant relation ( $\mathrm{p}$ value $>$ 0.05 ) between gender and serum TRAb and TPOAb, as shown in Table 2. The $\mathrm{p}$ value of all parameters, values statistically of less than 0.05 regarded as significant, are shown in Table 2.

An independent-samples t-test was run to determine the difference in means of serum TRAb between Graves' disease-diagnosed group versus Thyroiditis-diagnosed group.

The mean TRAb in Graves' disease group was $(10.38 \pm 8.910)$ compared to Thyroiditis group (1.14 \pm 2.813$)$, a statistically significant difference of 0.005 , (95\% CI, 6.23 to 12.19), $\mathrm{t}(88)=6.14, \mathrm{p}<0.005$, as shown in Table 3 .

The mean TPOAb in Graves' disease group was (30.654 \pm 30.054$)$ compared to Thyroiditis group ( $32.144 \pm 41.640)$. There was no statistically significant difference in mean TPOAb level in Graves' disease group and thyroiditis, with Graves' disease group TPOAb level with no statistically significant difference $=0.85$, $(95 \% \mathrm{CI},-17.19$ to 14.21$), \mathrm{t}(65.99)=-1.19, \mathrm{p}=0.85$.

Table 2. The significant values of different variables of the study.

\begin{tabular}{|c|c|c|c|c|}
\hline Variables & Number & Percent \% & $\begin{array}{c}\text { TRAb } \\
(\mathrm{p} \text { value }<0.05)\end{array}$ & $\begin{array}{c}\text { TPOAb } \\
(\mathrm{p} \text { value }<0.05)\end{array}$ \\
\hline & 120 & & & \\
\hline Age $($ Mean $\pm S D)$ & $37.97 \pm 12.00$ & & 0.428 & 0.5 \\
\hline Gender (M/F) & & & $<0.000$ & 0.001 \\
\hline Goiter & 89 & 74.2 & 0.418 & 0.00002 \\
\hline Weight Loss & 79 & 65.8 & 0 & 0.00004 \\
\hline Ophthalmopathy & 24 & 20 & $<0.0005$ & 0.001 \\
\hline Ultrasound: Diffuse & 67 & 55.8 & 0.01 & 0.672 \\
\hline TSH & & & 0.036 & 0.499 \\
\hline $\mathrm{fT} 4$ & & & 0.023 & 0.748 \\
\hline TPOAb & & & 0.395 & \\
\hline TRAb & & & & 0.395 \\
\hline Diagnosis of Groups & & & 0.0001 & 0.0502 \\
\hline
\end{tabular}

Table 3. Mean variance between Graves' disease and Thyroiditis groups.

\begin{tabular}{cccccccc}
\hline & T & Df & Sig. (2-tailed) & $\begin{array}{c}\text { Mean } \\
\text { Difference }\end{array}$ & $\begin{array}{c}\text { Std. Error } \\
\text { Difference }\end{array}$ & \multicolumn{2}{c}{$\begin{array}{c}\text { 95\% Confidence } \\
\text { Interval of the Difference }\end{array}$} \\
\hline Age & 0.83 & 89 & 0.41 & & & Lower & Upper \\
\hline TPOAb & 0.19 & 65.9 & 0.85 & -1.48974 & 7.86317 & -17.19 & 14.29 \\
TRAb & 6.14 & 88 & 0.000 & 9.208 & 1.315 & 6.226 & 12.19 \\
fT4 & 2.27 & 56.4 & 0.027 & 0.21154 & 0.09306 & 0.025 & 0.392 \\
TSH & -0.13 & 89 & 0.896 & -0.00641 & 0.0488 & -0.104 & 0.096 \\
\hline & & & & & & &
\end{tabular}


The fT4 in Graves' disease group was $(1.9038 \pm 0.29768)$ compared to Thyroiditis group $(1.692 \pm 0.521)$, a statistically significant difference $=0.027$, (95\% CI, 0.0251 to 0.398$), \mathrm{t}(56)=2.273, \mathrm{p}=0.027$.

The TSH in Graves' disease group was ( \pm 1.942$)$ compared to Thyroiditis group, a statistically no significant difference $=0.896$, (95\% CI, -0.10 to 0.09$), \mathrm{t}(89)=$ $-0.13, \mathrm{p}=0.89$, as shown in Table 3 .

\section{Discussions}

Graves' disease (autoimmune hyperthyroidism) is the commonest cause of thyrotoxicosis. A correct diagnosis of Graves' disease is important as treatment of thyrotoxicosis depends upon the cause. The committee agreed that it is crucial to determine the cause of thyrotoxicosis because this affects management decisions. TRAbs testing provides confirmation of clinical features that suggest Graves' disease [10].

In our study we found the importance of TRAb test in diagnosis of Graves' disease for differentiation from other non-Graves causes of hyperthyroidism. Using TRAb test we could diagnose, many cases of Graves, otherwise difficult to diagnose, depending on clinical findings and other investigations. We could differentiate cases of thyroiditis from Graves' disease, depending on negative TRAbs and other tests as TPOAbs and inflammatory markers such as ESR and CRP and ultrasound examination of thyroid gland. Thyroid scan with Tc99 perchlorate and Radio-active iodine scans were not available in our locality for diagnosis. As such there was much difficulty in differentiating diagnosis of many cases of thyrotoxicosis.

Evidence suggested that in adults, the diagnostic accuracy of TRAbs testing for Graves' disease was high across different cut-off values. Evidence also showed the accuracy to be high in children [11]. This study was in line with the committee's experience and current practice.

In our study we found that TPOAbs are not diagnostic test to differentiate between etiologies of thyrotoxicosis, and there was no significant difference in of TPOAb levels in Graves' disease group of patients and Thyroiditis group of patients in our study. The committee agreed that based on their experience, TPOAbs testing alone is not likely to be as useful as TRAbs testing for the diagnosis of Graves' disease, but it could be used in children and young people where the absence of TRAbs but presence of TPOAbs indicates that thyrotoxicosis is more likely to resolve spontaneously [11].

In our study, Ultrasound findings were not sensitive neither for differentiation between Graves' disease and thyroiditis, nor for diagnosis of Graves' disease per se, according to our results. The study is compatible with NICE guidelines recommendations. Evidence for the diagnostic accuracy of ultrasound was limited in both adults and children. Based on clinical experience, the committee agreed that ultrasound was useful for the diagnosis of Graves' disease but only when there were palpable thyroid nodules [11].

We couldn't use technetium scanning or radioactive iodine uptake in our study because of unavailability of the test in our locality, which makes the TRAb testing an essential diagnostic tool in this situation, for final diagnosis of Graves' 
disease and differentiating it with thyroiditis disease patients, and some cases of toxic nodular goiter.

The committee agreed that technetium scanning may be useful when TRAbs are negative and Graves' disease is suspected because generalized uptake on the scan suggests Graves' disease [11].

Areas with a low iodine intake tend to have a high incidence of toxic multinodular goiter (TMNG). Cases of GD could be misdiagnosed as TMNG in these areas if investigations other than radionuclide imaging are not considered. Although GD and TMNG can present concurrently, TRAb titers could be used to distinguish TMNG from GD [12].

TRAb tests are useful in distinguishing GD from subacute painless thyroiditis (SPT). The clinical presentation of SPT may be similar to GD, with thyrotoxicosis and a diffuse, non-nodular goiter. However, in SPT, the thyrotoxicosis is caused by autoimmune destructive phenomena rather than sustained hormone synthesis, and the condition is self-limited, resolving spontaneously over a few weeks. Almost all patients with SPT test positive for thyroid peroxidase antibodies, but so do up to $70 \%$ of patients with GD [13], so this excellent test for thyroid autoimmunity, in general, cannot distinguish the 2 conditions.

TRAb are also very useful in distinguishing postpartum thyroiditis from de novo or relapsing GD in lactating women, when RAIU+S must be avoided [14].

The TRAb titer is a cost-effective test in making the distinction between GD and subacute thyroiditis when the clinical presentation is ambiguous [15].

An interesting result of our study was the relatively high occurrence of TRAb in patients primarily classified as having MNTG (17\%). Only a few studies have included TRAb measurements in larger groups of MNTG patients [12].

Classical MNTG is a complication of multinodular goiter and the hyperthyroidism is due to hypersecretion of thyroid hormones from multiple autonomous nodules. However, if Graves' disease develops on top of a multinodular goiter, this may be difficult to separate from the classical type. This is especially a problem in areas with a low iodine intake such as ours, because of the high prevalence of nontoxic multinodular goiter [12].

The sensitivity and specificity of modern 3rd generation TRAb assays has significantly improved. The likelihood of a TRAb-positive individual to have GD was $>1000$ to $>3000$ fold greater (depending upon the type of assay) compared to a TRAb-negative person. Another study that compared the performance characteristics of different TBII and bioassays for TRAbs reported 100\% specificity for all the assays [16].

In our study we found strong relation between TRAb test positivity and $\mathrm{Di}$ agnosis of Graves' disease. There was strong correlation between TRAb test and serum TSH, fT4, TPOAbs, and gender in our study.

We couldn't depend on ultrasound examination for our diagnosis of Graves' disease and differentiating between Graves' disease and Thyroiditis, nor for diagnosis of thyroiditis in our study. 
Diana T. et al. compared the performance characteristics of different TBII and bioassays for TRAbs reported $100 \%$ specificity for all the assays [16]. Furthermore, in untreated hyperthyroid patients the sensitivity and specificity of 3rd generation TBII assays is of the order of $98 \%$ and $99 \%$, respectively [17].

Beastall G. et al. indicate that for a significant number of patients the correct diagnosis of GD or non-GD hyperthyroidism cannot be made on clinical presentation alone. A consequence of incorrect diagnosis may include unnecessary investigations, treatment and follow-up [8].

We didn't perform TRAb study for patients with toxic nodular goiter for the purpose of cost-effective concerns and in some situations, as unnecessity as inability of TRAb to differentiate between the two diagnosis, unless coexistence of the two disease at the same time. However, it is also advisable to perform TRAb test for TNG patients, first to find coexisting diseases in a number of patients who have both diseases, and also to further confirm the absence of Graves' disease in TNG patients.

Our study of correlation between the various degrees of manifestations and the level of TRAb at diagnosis corroborated the central role of TSH-receptor autoimmunity in Graves' disease. A clear positive correlation was observed between the concentration of TRAb in serum and first, thyroid volume, second, degree of biochemical hyperthyroidism, and third, the presence of eye signs [18].

Peter Laurberg et al. suggest that, there was correlation between fT4, TSH, TRAb study, while in other study "No correlation was found with TPO-Ab or any of the other variables investigated" [18].

Lauren Bell et al. have found that the TRAb result was the definitive diagnosis against which we evaluated the sensitivity and specificity of clinical diagnosis [7].

These findings suggest clinicians utilize a positive TRAb measurement over their clinical judgement to confirm a diagnosis of GD. A TRAb measurement in the initial clinical assessment of all patients could potentially prevent an incorrect diagnosis being made in the first place [7].

In the study, presence of orbitopathy was regarded as diagnostic feature for Graves' disease [18], and high titer of TRAb levels and strong correlation with Graves' disease subgroup, indicated high sensitivity and sensitivity test as indicated by other studies [1] [8] [15] [16].

\section{Conclusion}

The TRAb test can help reduce the number of incorrect or unknown diagnoses in the initial clinical assessment of patients presenting with hyperthyroidism. There was strong evidence to use TRAb as diagnostic tool in diagnosis of Graves' disease and differentiate it with different types of thyroiditis presenting with features of thyrotoxicosis, exclusively in areas of unavailability of scintiscan scanning for thyroid gland. There is also necessity for using TRAb in some cases presenting with features of toxic nodular goiter. Therefore, TRAb test is essential in most patients presenting with clinical features of hyperthyroidism, the diagnosis is clear and there is no need for further assessment. 


\section{Conflicts of Interest}

The author declares that there is no conflict of interests regarding the publication of this paper.

\section{References}

[1] Ross, D.S., Burch, H.B., Cooper, D.S., Greenlee, M.C., Laurberg, P., Maia, A.L., et al. (2016) American Thyroid Association Guidelines for Diagnosis and Management of Hyperthyroidism and Other Causes of Thyrotoxicosis. Thyroid, 26, 1343-1421. https://doi.org/10.1089/thy.2016.0229

[2] Berghout, A., Wiersinga, W.M., Smits, N.J. and Touber, J.L. (1990) Interrelationships between Age, Thyroid Volume, Thyroid Nodularity, and Thyroid Function in Patients with Sporadic Nontoxic Goiter. The American Journal of Medicine, 89, 602-608. https://doi.org/10.1016/0002-9343(90)90178-G

[3] Laurberg, P., Pedersen, K.M., Hreidarsson, A., Sigfusson, N., Iversen, E. and Knudsen, P.R. (1998) Iodine Intake and the Pattern of Thyroid Disorders: A Comparative Epidemiological Study of Thyroid Abnormalities in the Elderly in Iceland and in Jutland, Denmark. The Journal of Clinical Endocrinology \& Metabolism, 83, 765-769. https://doi.org/10.1210/jcem.83.3.4624

[4] Abraham-Nordling, M., Byström, K., Törring, O., Lantz, M., Berg, G., Calissendorff, J., et al. (2011) Incidence of Hyperthyroidism in Sweden. European Journal of Endocrinology, 165, 899-905. https://doi.org/10.1530/EJE-11-0548

[5] Roti, E., Minelli, R., Giuberti, T., Marchelli, S., Schianchi, C., Gardini, E., et al. (1996) Multiple Changes in Thyroid Function in Patients with Chronic Active HCV Hepatitis Treated with Recombinant Interferon-Alpha. The American Journal of Medicine, 101, 482-487. https://doi.org/10.1016/S0002-9343(96)00259-8

[6] Franklyn, J.A. and Boelaert, K. (2012) Thyrotoxicosis. The Lancet, 379, 1155-1166. https://doi.org/10.1016/S0140-6736(11)60782-4

[7] Bell, L., Hunter, A.L., Kyriacou, A., Mukherjee, A. and Syed, A.A. (2018) Clinical Diagnosis of Graves' or Non-Graves' Hyperthyroidism Compared to TSH Receptor Antibody Test. Endocrine Connections, 7, 504-510. https://doi.org/10.1530/EC-18-0082

[8] Beastall, G., Beckett, G., Franklyn, J., Fraser, W., Hickey, J., John, R., et al. (2006) UK Guidelines for the Use of Thyroid Function Tests.

[9] Smith, B.R., Sanders, J. and Furmaniak, J. (2007) TSH Receptor Antibodies. Thyroid: Official Journal of the American Thyroid Association, 17, 923-938. https://doi.org/10.1089/thy.2007.0239

[10] NICE. Thyroid Disease: Assessment and Management. https://www.nice.org.uk/guidance/ng145

[11] Recommendations. Thyroid Disease: Assessment and Management. https://www.nice.org.uk/guidance/ng145/chapter/recommendations

[12] Pedersen, I.B., Knudsen, N., Perrild, H., Ovesen, L. and Laurberg, P. (2001) TSH-Receptor Antibody Measurement for Differentiation of Hyperthyroidism into Graves' Disease and Multinodular Toxic Goitre: A Comparison of Two Competitive Binding Assays. Clinical Endocrinology, 55, 381-390. https://doi.org/10.1046/j.1365-2265.2001.01347.x

[13] Mariotti, S., Caturegli, P., Piccolo, P., Barbesino, G. and Pinchera, A. (1990) Antithyroid Peroxidase Autoantibodies in Thyroid Diseases. The Journal of Clinical Endocrinology \& Metabolism, 71, 661-669. https://doi.org/10.1210/jcem-71-3-661 
[14] Barbesino, G. and Tomer, Y. (2013) Clinical Utility of TSH Receptor Antibodies. The Journal of Clinical Endocrinology \& Metabolism, 98, 2247-2255. https://doi.org/10.1210/jc.2012-4309

[15] Matthews, D.C. and Syed, A.A. (2011) The Role of TSH Receptor Antibodies in the Management of Graves' Disease. European Journal of Internal Medicine, 22, 213-216. https://doi.org/10.1016/j.ejim.2011.02.006

[16] Diana, T., Wüster, C., Kanitz, M. and Kahaly, G.J. (2016) Highly Variable Sensitivity of Five Binding and Two Bio-Assays for TSH-Receptor Antibodies. Journal of Endocrinological Investigation, 39, 1159-1165. https://doi.org/10.1007/s40618-016-0478-9

[17] Tozzoli, R., Bagnasco, M., Giavarina, D. and Bizzaro, N. (2012) TSH Receptor Autoantibody Immunoassay in Patients with Graves' Disease: Improvement of Diagnostic Accuracy over Different Generations of Methods. Systematic Review and Meta-Analysis. Autoimmunity Reviews, 12, 107-113. https://doi.org/10.1016/j.autrev.2012.07.003

[18] Laurberg, P., Nygaard, B., Andersen, S., Carlé, A., Karmisholt, J., Krejbjerg, A., et al. (2014) Association between TSH-Receptor Autoimmunity, Hyperthyroidism, Goitre, and Orbitopathy in 208 Patients Included in the Remission Induction and Sustenance in Graves' Disease Study. Journal of Thyroid Research, 2014, Article ID: 165487. https://www.ncbi.nlm.nih.gov/pmc/articles/PMC3945164 https://doi.org/10.1155/2014/165487

\section{Abbreviations and Acronyms}

FT4: Free thyroxine

TSH: Thyroid stimulating hormone

TRAb: TSH Receptor Antibodies

TPOAb: Thyroid peroxidase Antibodies

RAIU: radioactive iodine uptake 